\title{
Vertebral artery dissection in hypertensive disorders of pregnancy: a case series and literature review
}

\author{
Renuka Shanmugalingam 13,5*, Nina Reza Pour', Siang Chye Chuah', Thi Mong Vo ${ }^{1}$, Roy Beran ${ }^{1}$,
} Annemarie Hennessy ${ }^{2,3,5}$ and Angela Makris, 1,3,5

\begin{abstract}
Background: Arterial dissection is a rare complication of pregnancy and puerperium. There have been reports of aortic, coronary and cervical artery dissection in association with preeclampsia, however, vertebral artery dissection is rarely reported particularly in the antenatal setting in the presence of a Hypertensive Disorder of Pregnancy (HDP).The general annual incidence of symptomatic spontaneous cervicocephalic arterial dissection is $0.0026 \%$ and a data registry reported that $2.4 \%$ of these occurred in the post-partum period. The actual incidence of vertebral artery dissection in HDP is unknown as the current literature consists of case series and reports only with most documenting adverse outcomes. Given the presence of collateral circulation, unilateral vertebral artery dissections may go unrecognised and may be more common than suspected.
\end{abstract}

Case presentation: We present a case series of four patients with vertebral artery dissection in association with HDP, two of which occurred in the antenatal setting and two in the post-partum setting. All our patients had favourable outcome with no maternal neurological deficit and live infants. Our discussion covers the proposed pathophysiology of vertebral artery dissection in HDP and the management of it.

Conclusion: Our case series highlights the need to consider VAD an important differential diagnosis when assessing pregnant women with headache and neck pain particularly in the context of HDP

Keywords: Hypertensive disorders of pregnancy, Vertebral artery dissection, Cerebrovascular accident, Hypertension

\section{Background}

Arterial dissection is a rare complication of pregnancy and puerperium. There have been reports of aortic, coronary and cervical artery dissection in association with preeclampsia, however, vertebral artery dissection (VAD) is rarely reported particularly in the antenatal setting in the presence of pre-eclampsia [1, 2]. Four cases of vertebral artery dissection are presented here in the context of hypertensive disorders of pregnancy (HDP). We report two cases of VAD which occurred in the antenatal period and two in the postpartum period. Our case series highlights the importance of recognizing VAD as a differential for occipito-cervical pain in women with HDP.

\footnotetext{
* Correspondence: renuka@hypercube3.com

'Liverpool Hospital, Elizabeth Street, Liverpool 2170, NSW, Australia

${ }^{3}$ Western Sydney University, Penrith, Australia

Full list of author information is available at the end of the article
}

\section{Case presentation}

\section{Case 1}

A 32 year old primigravid Fijian -Indian non-smoker, with no significant personal or family medical history presented at $38+2$ weeks gestation in early labour. Her initial blood pressure (BP) was 130/70 $\mathrm{mmHg}$ with reassuring cardiotocogram (CTG) trace. She reported a four day history of left neck pain which was described as dull and constant in the post auricular region with radiation down to the base of the neck with no neurological signs on examination. Urine analysis on presentation showed $3+$ of protein. Within two hours of presentation, she reported sudden onset of headache and dizziness with a recorded $\mathrm{BP}$ of $182 / 113 \mathrm{mmHg}$ and variable decelerations on CTG. She rapidly progressed on to an eclamptic fit despite oral and intravenous antihypertensive agents. 
Emergency lower segment caesarean section (LSCS) was performed under general anaesthetic and a male infant (weight $2460 \mathrm{~g}$ ) was delivered with normal placental histopathology. Retrospective sFlt- 1 (soluble fms-like tyrosine kinase 1) and PlGF (Placental Growth Factor ) concentrations (ELISA, R \& D) on serum sample obtained on presentation was $13,150 \mathrm{pg} / \mathrm{ml}$ and $190 \mathrm{pg} / \mathrm{ml}$ respectively (sFLT-1/ PIGF ratio: 69.2). Extubation occurred within $12 \mathrm{~h}$ and she reported ongoing neck pain. A computerized tomography angiogram (CTA) of her neck demonstrated a focal stenosis in the left vertebral artery with an intramural thrombus at the level of the third cervical vertebrae (C3) (Fig. 1a). This was further investigated with a magnetic resonance imaging scan (MRI) which confirmed a short segment VAD at C3 with no evidence of posterior circulation cerebral infarct (Fig. 1b and c). She was commenced on aspirin $100 \mathrm{mg}$ daily. A repeat brain and neck CTA at three months post-partum showed resolution of the dissection and aspirin was ceased. A vasculitic screen showed an antinuclear antibody (ANA) titre ratio of 1: 640 with a fine speckled fluorescent distribution. Her double stranded DNA, ENA and lupus anticoagulant were negative. She was initiated on Hydroxychloroquine for possible Systemic Lupus Erythematosis particularly with the onset of polyarthalgia in the postpartum setting.

\section{Case 2}

A 33-year-old primigravid Fijian-Indian non-smoker with no significant personal or family medical history attended a routine antenatal appointment at 36/40 weeks gestation and a BP of 150/110 $\mathrm{mmHg}$ was recorded. The spot urinary protein to creatinine ratio (uPCR) was $157.5 \mathrm{mg} / \mathrm{mmol}$ and a fetal ultrasound showed appropriate interval fetal growth with no evidence of placental insufficiency. She was initiated on regular oral antihypertensives and admitted for expectant management of pre-eclampsia. Day four of admission $(36+4 / 40)$, she reported rapid onset of right sided neck pain which started at the base of her neck and radiated to the post auricular region. This was concurrent with high diastolic BP readings of $90-110 \mathrm{mmHg}$ over $12 \mathrm{~h}$ with preserved systolic BP control (125 - $135 \mathrm{mmHg})$. Dose of regular antihypertensives were increased and an urgent MRI/MRA neck demonstrated a $1.1 \mathrm{~cm} \mathrm{C2-C3}$ right vertebral artery dissection with no evidence of posterior circulation cerebral infarct. No inciting event could be recalled. Emergency LSCS was performed and a female infant (weight $2900 \mathrm{~g}$ ) was delivered with a grossly normal placenta. She was transferred to high dependency unit post-operatively and initiated on a heparin infusion $6 \mathrm{~h}$ post-operatively. Within $24 \mathrm{~h}$, she developed progressive abdominal symptoms with haemodynamic compromise necessitating surgical re-exploration and evacuation of haematoma. Heparin was replaced by daily aspirin. A
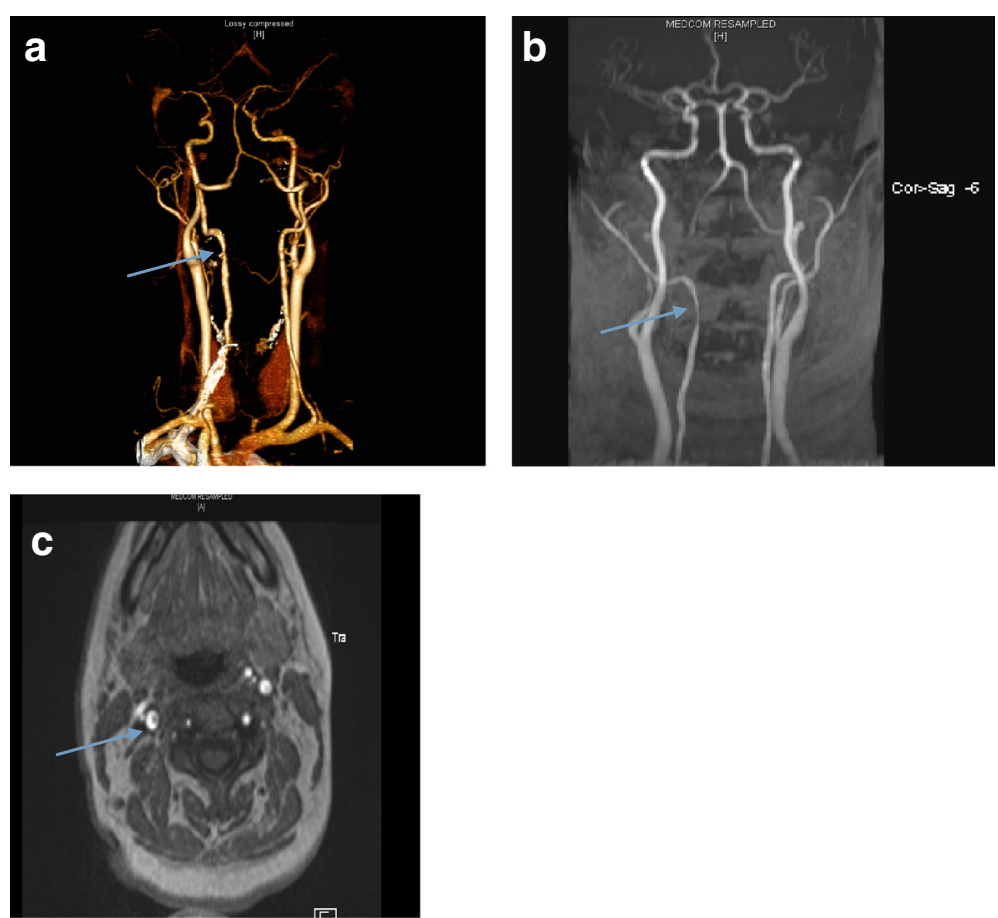

Fig. 1 a CTA Neck : Focal stenosis with thickened wall and an intramural thrombus of left vertebral artery at the level of C3 (arrow). b and c A MRI coronal (b) and horizontal (c) view of the vertebral arteries confirms a $1.1 \mathrm{~cm} \mathrm{C2-C3} \mathrm{right} \mathrm{vertebral} \mathrm{artery} \mathrm{dissection} \mathrm{with} \mathrm{no} \mathrm{evidence} \mathrm{of} \mathrm{posterior}$ circulation cerebral infarct (arrow) 
repeat CTA at three months showed resolved dissection and aspirin was ceased. Vasculitic screen was unremarkable.

\section{Case 3}

A 30-year-old Caucasian, gravida two para two, presented six days postpartum with two days history of headache, chest tightness, shortness of breath and bilateral pedal oedema. She was an obese $(B M I=32)$, nonsmoker, with a history of infrequent migraines and no other significant personal or family history. Her first pregnancy, three years prior, was uncomplicated but with different paternity. The antenatal and intrapartum care was uneventful and she delivered a $3800 \mathrm{~g}$ male infant at 39 weeks gestation. She was normotensive on discharge, three days later. Day six post-partum, she presented with the symptoms above and reported taking non-steroidal anti-inflammatory drugs (NSAIDs). BP at presentation was $180 / 110 \mathrm{mmHg}$ and neurological examination was unremarkable. CT pulmonary angiogram (CTPA) and CT Brain (CTB) were both negative for pulmonary embolism and intracerebral haemorrhage respectively. Two days into her admission, she reported ongoing headache with radiation down the right cervical region. Her blood pressure readings ranged between SBP of $130-140 \mathrm{mmHg}$ and DBP of $80-90 \mathrm{mmHg}$. She underwent a MRI brain which demonstrated an intramural thrombus at the level of $\mathrm{C} 2$ indicating a right VAD. Therapeutic dose of subcutaneous low-molecularweight heparin (LMWH) was commenced in addition to antihypertensive agents. MRA six weeks later demonstrated resolution of the vertebral artery intramural thrombus. LMWH was replaced by aspirin for an additional 6 weeks to complete a total of 3 months of therapy. Antihypertensive medications were ceased as her blood pressure normalized and a vasculitic screen was unremarkable.

\section{Case 4}

A 30-year-old non-smoking Pakistani, gravid 2 para 2, presented at 6 days post-partum with left sided neck pain within $24 \mathrm{~h}$ of discharge. Her first pregnancy was complicated by IUGR and post-partum eclampsia. In the second pregnancy, calcium supplement and low dose aspirin were commenced prior to 14 weeks gestation. Antenatal course was unremarkable with the same paternity and a female infant (weight $2720 \mathrm{~g}$ ) was delivered at 39 weeks gestation. Post-partum period was complicated by post-partum haemorrhage with disseminated intravascular coagulation (DIC) necessitating Ergometrin, intramyometrial Prostaglandin F2 Alpha and transfusion with blood product. She received a single dose of NSAIDs day 3 post-partum, following which her blood pressure was noted to be elevated (maximum 150/
$98 \mathrm{mmHg}$ ). Oral antihypertensive agents were commenced and she was discharged on day 5. She represented within $24 \mathrm{~h}$ with a BP of $155 / 95 \mathrm{mmHg}$ and pain at the left base of her neck with radiation to her ipsilateral shoulder without neurological deficit. A CTA demonstrated a left vertebral artery intramural thrombus at the level of $C 4 / 5$ for which she was commenced on aspirin $100 \mathrm{mg}$ daily. Her vasculitic screen was unremarkable and a repeat CTA 3 months later showed resolution of thrombus, after which aspirin was ceased.

\section{Discussion}

Vertebral artery dissection is a rare complication of HDP. The general annual incidence of symptomatic spontaneous cervicocephalic arterial dissection (CCAD) is $0.0026 \%$ and a data registry reported that $2.4 \%$ of CCAD occurred in the post-partum period [3, 4]. The actual incidence of VAD in association with HDP is unknown as the current literature consists of case series and reports only with most reporting adverse maternal outcomes (Table 1) [1, 2, 5]. We present two cases of VAD in the antenatal setting and two in the post-partum setting with good maternal and fetal outcomes. VAD is commonly associated with cervical manipulation, rapid head turning or cervical trauma and predisposing conditions includes Marfan's Syndrome, fibromuscular dysplasia and vasculitis [4]. Our first patient (Case 1) reported having had a neck massage 2 days after the onset of pain. There was no change in the characteristic of her pain following this. There is no known ethnicity predisposition to VAD, therefore, it is unclear if the association between VAD and the ethnicity of our patients (3 of 4 of them were of South - Asian ancestry) is coincidental or due to an underlying genetic predisposition. The common feature in all cases is the presence of a HDP. Two patients (Cases 1 \&2) had pre-eclampsia and the remaining two patients (Cases $3 \& 4$ ) had NSAIDs induced post-partum hypertension in an otherwise unremarkable antenatal course. The area of NSAIDs induced hypertension remains subject to ongoing study, though there have been suggestions of NSAIDs precipitating post-partum hypertension in patients at risk [6, 7]. Additionally, the use of Ergometrin and Prostaglandin F2 Alpha, smooth muscle contractors in case 4 could have contributed towards to surge in her previously normal blood pressure.

An imbalance in angiogenesis factors, Placental growth factor (PIGF), soluble FMS-like tyrosine kinase 1(sFlt-1) and soluble endoglin factor (sEng) is present in various degrees of severity in HDPs $[8,9]$. It is thought that this biochemical milieu that is well recognized to cause maternal endothelial dysfunction makes the endothelial layer susceptible to intimal rupture $[8,9]$. Hypertensive surge is possibly a risk factor for dissections and it is 
Table 1 Summary of our 4 cases with the case reports in the literature

\begin{tabular}{|c|c|c|c|c|c|c|c|c|}
\hline & Age & Risk factors & $\mathrm{HDP}$ & Symptom & Neurological signs & Event time & Treatment & Outcome \\
\hline Case 1 & 32 & None & $\begin{array}{l}\text { Yes - } \\
\text { Preeclampsia }\end{array}$ & Left sided neck pain & No & Antenatal & Aspirin & $\begin{array}{l}\text { Alive - no } \\
\text { neurological deficit }\end{array}$ \\
\hline Case 2 & 33 & None & $\begin{array}{l}\text { Yes - } \\
\text { preeclampsia }\end{array}$ & $\begin{array}{l}\text { Right sided neck } \\
\text { pain }\end{array}$ & No & Antenatal & $\begin{array}{l}\text { Heparin } \\
\text { - > Aspirin }\end{array}$ & $\begin{array}{l}\text { Alive - no } \\
\text { neurological deficit }\end{array}$ \\
\hline Case 3 & 30 & Migraine, Obesity & $\begin{array}{l}\text { Yes - NSAID } \\
\text { induced } \\
\text { postpartum HTN }\end{array}$ & $\begin{array}{l}\text { Headche with left } \\
\text { sided neck pain }\end{array}$ & No & Postnatal & $\begin{array}{l}\text { LMWH } \\
->\text { Aspirin }\end{array}$ & $\begin{array}{l}\text { Alive - no } \\
\text { neurological deficit }\end{array}$ \\
\hline Case 4 & 30 & $\begin{array}{l}\text { Previous IUGR and } \\
\text { post partum } \\
\text { eclampsia }\end{array}$ & $\begin{array}{l}\text { Yes - NSAID } \\
\text { induced } \\
\text { postpartum HTN }\end{array}$ & Left sided neck pain & No & Postnatal & Aspirin & $\begin{array}{l}\text { Alive - no } \\
\text { neurological deficit }\end{array}$ \\
\hline $\begin{array}{l}\text { McKinney J } \\
\text { et al. [1] }\end{array}$ & 41 & None & Yes & $\begin{array}{l}\text { Headache, HTN, } \\
\text { Dysarthria }\end{array}$ & Quadriplegia & Post natal & Aspirin & $\begin{array}{l}\text { Alive with residual } \\
\text { memory and visual } \\
\text { loss }\end{array}$ \\
\hline \multirow{5}{*}{$\begin{array}{l}\text { Arnold M } \\
\text { et al. [2] }\end{array}$} & 41 & Migraine & - n/a- & Bilateral neck pain & TIA & Post natal & $-n / a-$ & \\
\hline & 35 & $\begin{array}{l}\text { Migraine, Smoker, } \\
\text { HTN }\end{array}$ & $-n / a-$ & Bilateral headache & Ischemic CVA & Postnatal & $-n / a-$ & Alive, Ischemic CVA \\
\hline & 27 & Migraine & $-n / a-$ & Ipsilateral neck pain & & Postnatal & $-n / a-$ & \\
\hline & 38 & Migraine, HTN & $-n / a-$ & $\begin{array}{l}\text { Thunderclap } \\
\text { headache }\end{array}$ & & Postnatal & $-n / a$ & \\
\hline & 34 & $\begin{array}{l}\text { Chiropractor neck } \\
\text { manipulation }\end{array}$ & $-n / a-$ & $\begin{array}{l}\text { Ipsilateral neck pain } \\
\text { with headache }\end{array}$ & & Postnatal & $-n / a-$ & \\
\hline $\begin{array}{l}\text { Tuluc M } \\
\text { et al. [5] }\end{array}$ & 39 & None & Yes & $\begin{array}{l}\text { Headache } \\
\text { preceeding loss of } \\
\text { consciousness }\end{array}$ & LOC & Antenatal & $-n / a-$ & $\begin{array}{l}\text { Deceased mother, } \\
\text { Live baby }\end{array}$ \\
\hline $\begin{array}{l}\text { Borelli P } \\
\text { et al. [3] }\end{array}$ & 34 & None & Yes & $\begin{array}{l}\text { Headache with visual } \\
\text { disturbance }\end{array}$ & None & Postnatal & Aspirin & Alive \\
\hline
\end{tabular}

thought that the vertebral artery is prone to the mechanical damage of hypertensive surge given its known vulnerability to traumatic injury $[10,11]$. In keeping with that, we observed that the onset of symptoms in our patients was closely related to a preceding surge in blood pressure. We therefore think that the combination of maternal endothelial dysfunction in HDP and the endothelial damaging effect of hypertensive surge potentially increases the risk of dissection in the naturally vulnerable vertebral artery. Passage of blood into this false lumen forces the intimal-medial layer towards the true lumen of the vessel, causing partial or full obstruction of flow, potentially leading to a cerebral ischaemic event $[4,12,13]$.

Headache in patients with HDP consist of a broad range of differential diagnosis from benign causes such as migraine to sinister causes such as Posterior Reversible Encephalopathy Syndrome (PRES), Intracerebral haemorrhage, Cerebral venous thrombosis, CCAD and impending eclampsia [14-17]. The incidence of headache in normal pregnancy, however, has been reported to range between $39 \%$ to 59 \% hence, creating a clinical dilemma in deciding who should be investigated particularly in the context of HDP [18]. There current recommendation is to investigate headache in pregnant women who have accompanying focal neurology features and those with headache resistant to analgesics though there is no data to support this practice $[17,19]$. We investigated our patient in Case 1 for CCAD given the ongoing occipito-cervical pain unrelieved by analgesics. We've since had a low threshold in investigating women with HDP who present with headache and neck pain unrelieved by simple analgesics. This led to the identification of VAD in the remaining 3 cases. We suspect that the occurrence of VAD in association with a hypertensive surge in HDP is more common than recognized and should be considered a differential diagnosis when assessing women with headache and neck pain particularly in the context of HDP.

A recent randomised trial showed no difference in efficacy between antiplatelet and anticoagulation in preventing stroke in patients with symptomatic CCAD [20]. There, however, remains a lack of consensus on the need for anticoagulation or antiplatelet altogether as some argue that the risk of secondary cerebrovascular event is low and would not warrant prophylactic therapy. Based on the data and our experience, we, would recommend the use of antiplatelet therapy in the immediate postpartum period given the risk of complications (Case2).

The actual incidence of recurrence of CCAD in subsequent pregnancies is unknown however prophylactic antiplatelet or antithrombotic therapy through the pregnancy 
would be advisable. There is currently no evidence on the superiority of one over the other in their use prophylactically in this setting. Aspirin has been shown to reduce the risk of pre-eclampsia in high risk women and thus would have a role in subsequent pregnancies of these women independent of the risk reduction of VAD [21].

\section{Conclusion}

Our case series highlights the need to consider VAD an important differential diagnosis when assessing women with headache and neck pain particularly in the context of HDP. Based on our experience, we would recommend investigating women with $\mathrm{HDP}$ who present with head or occipito-cervical pain for VAD particularly in the setting of a hypertensive surge. This is particularly important in the antenatal setting as theoretically, expulsive efforts in labor could risk extending the dissection and therefore VAD in the antenatal setting would warrant a multidisciplinary approach in deciding on a safe mode of delivery [22].

\section{Abbreviations}

ANA, Antinuclear antibody; BMI, Body mass index; BP, Blood Pressure; CTG, Cardiotocography; CCAP, Cervicocephalic arterial dissection;CTA, Computed Tomography Angiography; DIC, Disseminated intravascular coagulation; ENA, Extractable Nuclear Antigen Antibody; HDP, Hypertensive disorders of Pregnancy; IUGR, Intrauterine growth retardation; LMWH, Low Molecular Weight Heparin; LSCS, Lower segment caesarean section; MRI, Magnetic Resonance Imaging; NSAIDs, Nonsteroidal anti-inflammatory drugs; PIGF, Placenta growth factor; PRES, Posterior reversible encephalopathy; s-FLT-1, Soluble fms-like tyrosine kinase - 1;SEng, Soluble endoglin factor; VAD, Vertebral artery dissection

\section{Acknowledgements}

None.

\section{Funding}

None.

\section{Availability of data and material}

Not applicable for this case series.

\section{Authors' contribution}

RS and NR contributed equally in the literature review, drafting of the manuscript and clinical management of the patients. Therefore, should be both regarded the first authors. SCC,TMV,RB,AH,AM contributed towards the clinical management, conceptualisation and manuscript preparation. All authors have read and approved the final version of the manuscript.

\section{Competing interests}

The authors declare that they have no competing interests.

\section{Consent for publication}

Written and verbal consent for publication was obtained from all four patients. Copies of the consent form are available for review by the Editor of this journal.

\section{Ethics approval and consent to participate}

No ethics approval was required as this is case series. Written and verbal consent was obtained from all four patients discussed in this case series.

\section{Author details}

'Liverpool Hospital, Elizabeth Street, Liverpool 2170, NSW, Australia. ${ }^{2}$ Campbelltown Hospital, Therry Road, Campbelltown 2560, NSW, Australia. ${ }^{3}$ Western Sydney University, Penrith, Australia. ${ }^{4}$ University of New South Wales, Sydney, Australia. ${ }^{5}$ Vascular Immunology Research Group, Heart Research Institute, Newtown, Sydney, Australia.
Received: 9 November 2015 Accepted: 9 July 2016

Published online: 16 July 2016

\section{References}

1. McKinney JS, et al. Intracranial vertebrobasilar artery dissection associated with postpartum angiopathy. Stroke Res Treat. 2010;2010:320627. doi: $10.4061 / 2010 / 320627$

2. Arnold M, et al. Postpartum cervicocephalic artery dissection. Stroke. 2008; 39(8):2377-9.

3. Borelli $P$, et al. Bilateral thalamic infarct caused by spontaneous vertebral artery dissection in pre-eclampsia with HELLP syndrome: a previously unreported association. J Stroke Cerebrovasc Dis. 2012;21(8):914. e9-10.

4. Park KW, et al. Vertebral artery dissection: natural history, clinical features and therapeutic considerations. J Korean Neurosurg Soc. 2008;44(3):109-15.

5. Tuluc M, Brown D, Goldman B. Lethal vertebral artery dissection in pregnancy: a case report and review of the literature. Arch Pathol Lab Med. 2006;130(4):533-5.

6. Makris A, Thornton C, Hennessy A. Postpartum hypertension and nonsteroidal analgesia. Am J Obstet Gynecol. 2004;190(2):577-8.

7. Ghuman N, et al. Hypertension in the postpartum woman: clinical update for the hypertension specialist. J Clin Hypertens (Greenwich). 2009:11(12):726-33.

8. Powe CE, Levine RJ, Karumanchi SA. Preeclampsia, a disease of the materna endothelium: the role of antiangiogenic factors and implications for later cardiovascular disease. Circulation. 2011;123(24):2856-69.

9. Levine RJ, et al. Soluble endoglin and other circulating antiangiogenic factors in preeclampsia. N Engl J Med. 2006;355(10):992-1005.

10. Kobayashi $\mathrm{H}$, et al. Extracranial and intracranial vertebral artery dissections: A comparison of clinical findings. J Neurol Sci. 2016;362:244-50.

11. Eguchi $\mathrm{K}$, et al. A case of vertebral artery dissection associated with morning blood pressure surge. Hypertens Res. 2005:28(10):847-51.

12. Cenkowski $\mathrm{M}$, et al. Spontaneous dissection of the coronary and vertebral arteries post-partum: case report and review of the literature. BMC Pregnancy Childbirth. 2012;12:122.

13. Riskin-Mashiah S, et al. Cerebrovascular reactivity in normal pregnancy and preeclampsia. Obstet Gynecol. 2001;98(5 Pt 1):827-32.

14. Sperling JD, et al. The role of headache in the classification and management of hypertensive disorders in pregnancy. Obstet Gynecol. 2015; 126(2):297-302.

15. Friedman EA. The relationship between headache and preeclampsia. Eur J Obstet Gynecol Reprod Biol. 2006;125(1):146-7.

16. Facchinetti $F$, et al. The relationship between headache and preeclampsia: a case-control study. Eur J Obstet Gynecol Reprod Biol. 2005;121(2):143-8.

17. Macgregor EA. Headache in pregnancy. Continuum (Minneap Minn). 2014 20(1 Neurology of Pregnancy):128-47.

18. Aegidius $\mathrm{K}$, et al. The effect of pregnancy and parity on headache prevalence: the Head-HUNT study. Headache. 2009;49(6):851-9.

19. Ramchandren S, Cross BJ, Liebeskind DS. Emergent headaches during pregnancy: correlation between neurologic examination and neuroimaging AJNR Am J Neuroradiol. 2007:28(6):1085-7.

20. investigators, C.t, et al. Antiplatelet treatment compared with anticoagulation treatment for cervical artery dissection (CADISS): a randomised trial. Lancet Neurol. 2015;14(4):361-7.

21. Voelker R. USPSTF: Low-dose aspirin may help reduce risk of preeclampsia. JAMA. 2014:311(20):2055.

22. O'Rourke N, Wollman L, Camann W. Bilateral spontaneous vertebral artery dissection: management during labor and vaginal delivery. Int J Obstet Anesth. 2004;13(1):44-6. 